\title{
Historia de la robótica: de Arquitas de Tarento al robot Da Vinci (Parte I)
}

\author{
Sánchez Martín FM, Millán Rodríguez F, Salvador Bayarri J, Palou Redorta J, \\ Rodríguez Escovar F, Esquena Fernández S, Villavicencio Mavrich H.
}

Servicio de Urología. Fundació Puigvert. Barcelona.

Actas Urol Esp. 2007;31(2):69-76

"Los únicos errores reales son los errores humanos" (Última Ley de la Robótica. Anónimo)

\section{RESUMEN}

HISTORIA DE LA ROBÓTICA: DESDE ARQUITAS DE TARENTO HASTA EL ROBOT DA VINCI. (PARTE I)

La cirugía robótica es la última novedad tecnológica en urología. Para conocer como funcionan los nuevos robots es interesante conocer su historia. La fabricación de máquinas que imitan al ser humano se ha mantenido desde hace más de 4000 años. Existen referencias a King-su Tse, China clásica, que inventa un autómata en el 500 a. C. Arquitas de Tarento (hacia 400 a.C.) es considerado el padre de la ingeniería mecánica, y uno de los maestros clásicos de la robótica occidental. Figuras como Heron de Alejandría, Hsieh-Fec, Al-Jazari, Roger Bacon, Juanelo Turriano, Leonardo da Vinci, Vaucanson o von Kempelen construyeron robots en la edad media, el renacimiento y el clasicismo. En el siglo XIX existe un auge de los autómatas y se producen importantes avances en todas las ramas de la ingeniería. En 1942 Asimov publica las tres leyes de la robótica coincidiendo con el inicio de la robótica moderna, basada en los avances en mecánica, electrónica e informática. El desarrollo de robots en el terreno industrial, bélico y aeroespacial durante el siglo XX permite la aparición de robots de gran precisión, útiles en cirugía, como el robot quirúrgico da Vinci (Intuitive Surgical Inc, Sunnyvale, CA, USA).

Palabras clave: Robótica. Urología robótica. Historia de la robótica. Historia de la medicina. Arquitas.

ABSTRACT

HISTORY OF ROBOTICS: FROM ARCHYTAS OF TARENTUM UNTIL DA VINCI ROBOT. (PART I)

Robotic surgery is the newst technologic option in urology. To understand how new robots work is interesting to know their history. The desire to design machines imitating humans continued for more than 4000 years. There are references to King-su Tse (clasic China) making up automaton at 500 a. C. Archytas of Tarentum (at around 400 a.C.) is considered the father of mechanical engineering, and one of the occidental robotics classic referents. Heron of Alexandria, Hsieh-Fec, Al-Jazari, Roger Bacon, Juanelo Turriano, Leonardo da Vinci, Vaucanson o von Kempelen were robot inventors in the middle age, renaissance and classicism. At the $\mathrm{XIX}^{\text {th }}$ century, automaton production underwent a peak and all engineering branches suffered a great development. At 1942 Asimov published the three robotics laws, based on mechanics, electronics and informatics advances. At $\mathrm{XX}^{\text {th }}$ century robots able to do very complex self governing works were developed, like da Vinci Surgical System (Intuitive Surgical Inc, Sunnyvale, CA, USA), a very sophisticated robot to assist surgeons.

Keywords: Robotics. Robotics in urology. History of medicine. History of robotics. Architas.

$\mathrm{L}$ a imagen del robot como máquina a semejanza con el ser humano ha prevalecido en las culturas desde hace muchos siglos. El afán por fabricar máquinas capaces de realizar tareas inde- pendientes ha sido una constante en la historia, a través de la que se han descrito infinidad de ingenios, antecesores directos de los actuales robots ${ }^{1}$. El futuro de la cirugía estará muy ligado a los 
robots, ingenios en los que el ser humano ha volcado su inventiva desde la antigüedad.

El término "robot" fue utilizado por primera vez por Karel Capek (en su obra de teatro "R.U.R." (“Rossum's Universal Robots", en español "Los robots universales del Rossum") ${ }^{2}$ (escrita en colaboración con su hermano Josef) y publicada en 1920. La palabra "robot" viene del vocablo checo "robota" que significa "trabajo"3, en el sentido de la obligatoriedad, entendido como servidumbre, trabajo forzado o esclavitud, en referencia sobre todo a los llamados "trabajadores alquilados" que vivieron en el Imperio Austrohúngaro hasta 1848. Este concepto entronca con la terminología "amo-esclavo" de los robots actuales, cuando las unidades basan cada movimiento en una orden humana. En "R.U.R." se desarrolla el concepto de la fabricación en línea ejecutada por robots humanoides, tanto desde el punto de vista narrativo como filosófico. Años más tarde la novela fue adaptada al cine en la película "Metrópolis" y el término robot quedó fijado para ese significado. Aunque los robots de Capek eran humanos artificiales orgánicos, la palabra robot es casi siempre utilizada para referirse a humanos mecánicos. El término androide puede referirse a cualquiera de éstos, mientras que un cyborg ("organismo cibernético" u "hombre biónico") es una criatura combinación de partes orgánicas y mecánicas (Fig. 1).

\section{Historia de la robótica antigua.}

Hacia el año 1300 a. C., Amenhotep, hijo de Hapu, hace construir una estatua de Memon, rey de Etiopía, que emite sonidos cuando la iluminan los rayos del sol al amanecer. Los egipcios desarrollaron modelos matemáticos muy avanzados y construyeron automatismos muy sofisticados, como el reloj de agua. Se tiene constancia de la existencia del ábaco ya entre el año 1000 y 500 a d C, aunque existen dudas sobre si fue en Babilonia o en China dónde fue inventado. Este ingenio matemático permitió el desarrollo de la computación y la inteligencia artificial que fueron desarrollándose paralelamente al interés por los automatismos y el diseño de máquinas imitadoras del ser humano. En la mitología judeocristiana, reflejada en la Biblia, Adán puede ser considerado por como el primer autómata de la histo-

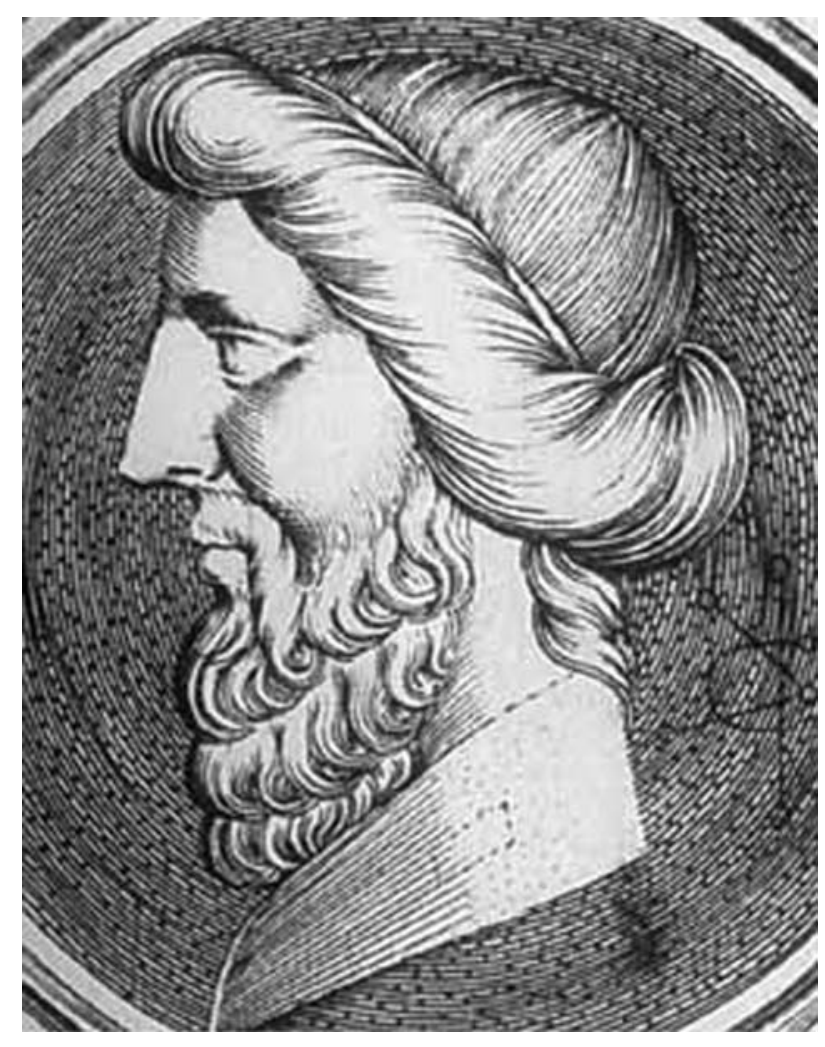

FIGURA 1. Arkitas de Tarento, uno de los primeros constructores de máquinas automáticas de la historia.

ria. Dios lo creó a partir de un material moldeable (barro), después lo programó y le dio las primeras instrucciones ${ }^{4}$. La mitología griega contiene asimismo referencias sobre la vida artificial: Prometeo creó el primer hombre y la primera mujer con barro y les dio vida mediante el fuego celeste. Pigmalión, rey y sacerdote mitológico, descubrió como Galatea, una de las estatuas que había esculpido, tomaba vida, desvelando con ello el interés atávico del ser humano por los autómatas. Ovidio lo narra así en su mitología: "Pigmalión se dirigió a la estatua y, al tocarla, le pareció que estaba caliente, que el marfil se ablandaba y que, deponiendo su dureza, cedía a los dedos suavemente...creyendo que se engañaba volvió a tocar la estatua otra vez, y se cercioró de que era un cuerpo flexible y que las venas daban sus pulsaciones..." En la mitología romana, Vulcano construye ingenios mecánicos que utiliza como sirvientes; mientras que en la hebrea el Gólem cobra vida con una combinación de palabras en el contexto de la magia cabalística. En la mitología nórdica el gigante Mökkurkálfi o 
Mistcalf fue construido para ayudar al troll Hrungnir en su duelo con Thor ${ }^{5}$.

King-su Tse, en China, inventa en el 500 a. C. una urraca voladora de madera y bambú, y un caballo de madera capaz de dar saltos ${ }^{6}$. Hacia el año 200 a. C., Filón de Bizancio, inventor de la catapulta repetitiva, construye un autómata acuático ${ }^{7}$. En el año 206 a. C., durante el reinado del primer emperador Han, fue encontrado el tesoro de Chin Shih Hueng Ti consistente en una orquesta mecánica de muñecos que se movian de forma independiente. En la antigua Grecia, Arquitas de Tarento (referenciado en inglés como Archytas of Tarentum ${ }^{8}$, y en algunas referencias en español como Architas de Tarento9), filósofo, matemático y político coetáneo de Platón ${ }^{10}$, considerado el padre de la ingeniería mecánica y precursor occidental de la robótica, inventó el tornillo y la polea, entre otros muchos dispositivos. Fabricó el primer cohete autopropulsado de la historia, que usó con fines militares. Hacia el año 400 a. C. construyó un autómata consistente en una paloma de madera que rotaba por si sola gracias a un surtidor de agua o vapor y simulaba el vuelo ${ }^{11}$. Hacia el 300 a. C., Cresibio (o Ctesibio ${ }^{12}$ o Ktesibios $^{13}$ ) inventa un reloj de agua (o clepsidra) y un órgano que emite los sonidos por impulsos de agua $^{14}$. Su amigo Filón de Bizancio inventa en el año 200 a. C., un autómata acuático y una catapulta automática ${ }^{15}$. En el año 62 de nuestra era Heron de Alejandría muestra, en su libro "Automata", los diseños de juguetes capaces de moverse por si solos de forma repetida, como aves que vuelan, gorjean y beben; o ingenios que funcionaban a partir de la fuerza generada por aspas de molino o circuitos de agua en ebullición, precursores rudimentarios de la turbina de vapor ${ }^{16}$. También se diseñan ingeniosos mecanismos como la máquina de fuego que abría puertas de los templos o altares mágicos donde las figuras apagaban el fuego de la llama. En la Roma imperial existía gran afición a los autómatas, que se exhibían en fiestas particulares, como el banquete de Trimalco, en el que un frutero presidido por un príapo arrojaba un chorro de perfume cuando se ejercía una ligera presión ${ }^{17}$. En año 335 d. C., Hsieh Fec construye un Buda montado en un carro de cuatro ruedas que se desplaza sin ayuda. En Oriente se producen asimismo algunas aportaciones $^{18}$ : El año 700 d. C., Huang Kun construye varias figuras animales y humanas que cantan y danzan ${ }^{6}$. En el 770 d. C., Yang Wu-Lien construye un mono que alarga las manos y grita “iLimosna!, guardando su recaudación en una bolsa cuando alcanza un peso determinado. El príncipe Kaya construye en el año 840 una muñeca que derrama agua. En el 890, Han Chih Ho presentó un gato de madera que cazaba ratas. En el año 1050 el príncipe hindú Bhoja escribe el Samarangana-Sutradhara, que incluye comentarios sobre la construcción de yantras, máquinas capaces de actuar por sí solas. En el siglo XII Al-Jazari (o Al-Djazari) ${ }^{6}$ construyó autómatas musicales impulsados por agua, con aplicaciones en la cocina y también fue un importante constructor de relojes de agua ${ }^{19}$. Los árabes, además del reloj, idearon diversos automatismos, como las máquinas dispensadoras de agua, recogiendo la herencia grecorromana y asiática, en busca no sólo de diversión sino de utilidad en tareas laborales.

Los relojes pueden considerarse como las máquinas antiguas más perfectas, muy cercanas al concepto de automatismo y, consecutivamente, a la de robótica. Es frecuente hallar relojes que incluyen figuras humanas móviles que se mueven con el orden de las horas. El reloj de la catedral de Munich y el reloj del Ánker de Viena son buenos ejemplos. El Gallo de Estrasburgo, el robot más antiguo que se conserva en la actualidad, funcionó desde 1352 hasta 1789. Formaba parte del reloj de la catedral y, al dar las horas, movía el pico y las $\operatorname{alas}^{20}$. En España, el Papamoscas de la catedral de Burgos, construido en el siglo XVI, consiste en un hombre mecánico que se mueve con los cambios horarios y funciona aún hoy día (Fig. 2).

Los autómatas más famosos del medioevo son el hombre de hierro de Alberto Magno (12041282) o la cabeza parlante de Roger Bacon (1214$1294)^{6}$. En el año 1235, Villard d'Honnecourt, constructor de la "rueda perpetua"21, escribe un libro con bocetos que incluyen secciones de dispositivos mecánicos antropomórficos, como un ángel autómata ${ }^{22}$. Leonardo da Vinci (1452-1519) construyó para el rey Luis XII de Francia un León Mecánico, que se abría el pecho con la garra y mostraba el escudo de armas real ${ }^{23}$. En 1495 ya 


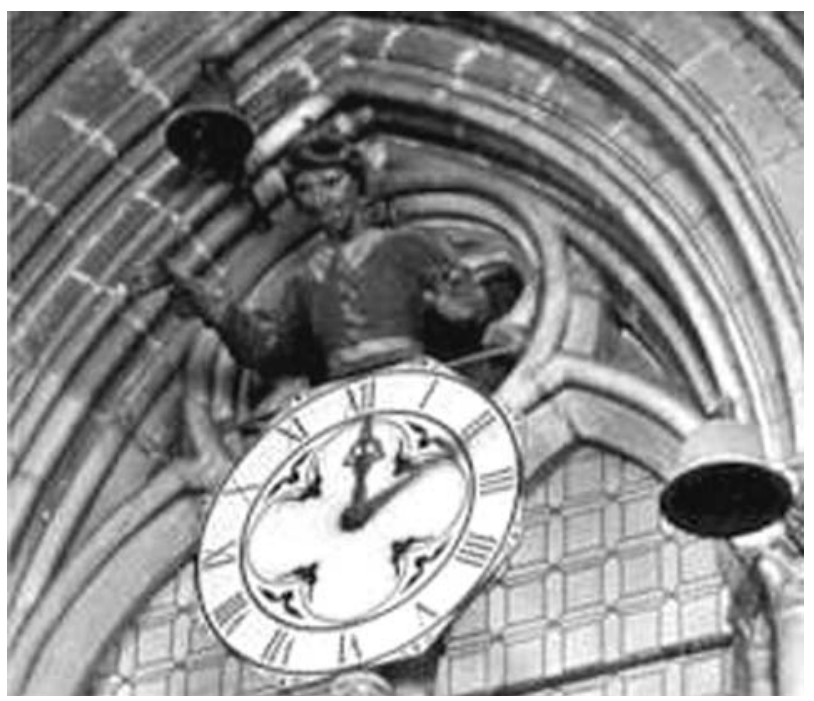

FIGURA 2. El papamoscas, autómata de la catedral de Burgos que se acciona con la coincidencia horaria.

había diseñado uno de los primeros autómatas humanoides del mundo occidental: un caballero con armadura, capaz de incorporarse, agitar los brazos, mover la cabeza (tenía un cuello flexible) y abrir y cerrar la mandíbula. Estas máquinas se relacionan con el canon anatómico del hombre vitruviano y sus claves matemáticas. Alrededor del año 1500 diseñó también una máquina de cálculo, predecesora de la que Blaise Pascal inventaría más de un siglo después por lo que el genio italiano proyectó la robótica desde el punto de vista formal y computacional ${ }^{24}$. Salomón de Caux (o Caus) (1576-1626), estudioso del vapor como fuente de energía, construyó diversos automatismos tomando como base la jardinería (fuentes, pájaros) ${ }^{25}$. A inicios del siglo XVI Hans Bullmann fabricó una pequeña orquesta de autómatas; en 1533 Johann Müller, conocido como Regiomontanus, construyó en Nuremburg varios pájaros voladores de metal y madera; y diez años más tarde John Dee presentó en Inglaterra su escarabajo de madera capaz de levantar el vuelo ${ }^{11}$.

En la España del siglo XVI Juanelo Turriano (originalmente Giovanni Torriani) (1500?${ }_{1585)^{26}}$, relojero del emperador Carlos V, construye el "Hombre de Palo", un monje autómata capaz de andar y mover la cabeza ${ }^{27}$. Salomón de Camus (o Camus le Lorrain) ${ }^{28}$ (1576-1626) construyó un coche en miniatura con caballos, lacayos y una dama en su interior, que se movian de forma armónica ${ }^{17}$. En 1640, René Descartes (15961650) construyó una autómata a la que llamaba "mon fill Francine", en memoria a su hija ${ }^{29}$. En 1662 tiene lugar la inauguración del teatro de autómatas Takedo en Osaka ${ }^{6}$. En el siglo XVII GW von Leibniz (1646-1716) abogó por el empleo del sistema binario como base para el cálculo automático, sentando definitivamente las bases de la computación actual ${ }^{30}$. Los materiales empleados para la construcción de autómatas eran la madera (partes formes), el hierro (estructura fija, soportes, goznes), el cobre (que es moldeable y permite construir partes más finas), el cuero (cables, calzado) y los tejidos. Los primeros modelos utilizaban la aplicación de fuerza directa para realizar los movimientos, facilitados con juegos de poleas, engranajes y palancas. En esta fase los autómatas eran réplicas del ser humano que realizaban una serie de movimientos simples. Las máquinas fueron asumiendo tareas de ayuda al hombre y acabaron repercutiendo en la propia concepción del mundo y de los seres animados. El mecanicismo afectó al estudio de la naturaleza, extendiéndose a la ciencia antómica, de la que se elaboraron modelos acordes con esa concepción, como puede ser el De Humanis Corporis Fabrica de Andrés Vesalio (o Andreas Vesalius) (1514-1564) que concibe al hombre como una compleja estructura mecánica ${ }^{31}$.

El desarrollo de la inteligencia artificial fue paralelo al de la invención de autómatas. Destacan con sus máquinas calculadoras John Napier (1550-1617) (1621), Wilhem Schickard (1592-1635) y Charles Babbage (1791-1871). Por su parte Leonhard Euler (1707-1783), Allen Marquand (1853-1924) y John Venn (1834-1923) trabajaron en algoritmos lógicos ${ }^{32}$. El desarrollo de modelos matemáticos con operativa mecánica, como el de George Boole (1815-1864), permitió pasar de la robótica clásica a la moderna tomando la computación como base ${ }^{33}$.

Jacques Vaucanson (o de Vaucanson) ${ }^{34}$ (17091782) es uno de los más famosos y completos constructores de androides automatizados de la historia $^{35}$. Persona de gran ingenio recorrió toda Europa presentando sus artefactos en las cortes de época. En 1738 montó un autómata flautista capaz de ejecutar melodías barrocas. El muñeco 
realizaba la digitación sobre el instrumento y seguía con los ojos la partitura. Además consiguió uno de los hitos más sonados de la historia de la robótica al construir un pato mecánico de más de 400 piezas móviles, capaz de graznar y comer de la mano del público, completando de forma total la digestión (Fig. 3). En el museo de autómatas de Grenoble existe una copia del pato de Vaucanson. Otros de los referentes de la robótica clásica son el relojero suizo Pierre JaquetDroz (1721-1790) ${ }^{36}$ y su hijo Henri-Louis que construyeron maquetas con paisajes animados (1774) -el más famoso fue el conocido como "la cueva"- y diversos muñecos capaces de escribir (1770), dibujar (1772) y tocar melodías a flauta o en un órgano (1773), entre los que destaca una mujer intérprete, cuya réplica se conserva en el museo de Neuchatel ${ }^{37}$. Puede accederse por Internet a las melodías para instrumentos de viento y piano ejecutadas por estos autómatas ${ }^{35}$. Jean-Frédéric Leschot, relojero y socio de los Jaquet-Droz, se especializó en la fabricación de prótesis para sustituir miembros amputados, consiguiendo no sólo cubrir el aspecto estético sino desarrollar un alto grado de funcionalidad para los brazos y piernas construidos ${ }^{38}$. En 1795 le encargan desde Francfort un brazo izquierdo para la baronesa Strakham. Al encargo, Leschot contesta: "Soy, gracias a Dios, capaz de hacer lo pedido. Varios objetos de esta naturaleza me han

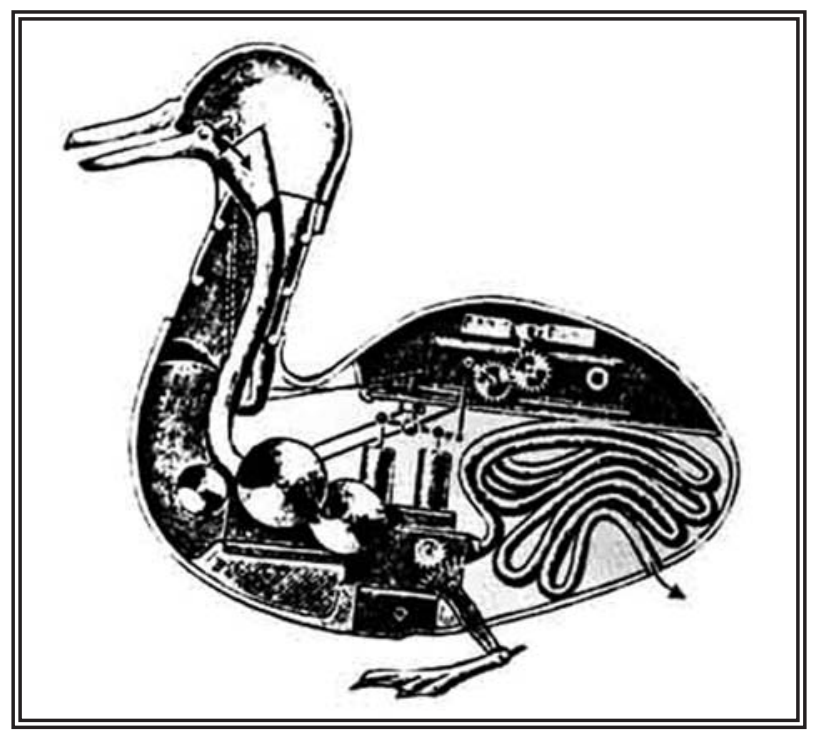

FIGURA 3. El pato de Vaucanson, uno de los ingenios mecánicos más famosos de la antigüedad. sido confiados y siempre salí airoso con la aprobación y el alivio de mis clientes. Hace unos años hice un brazo postizo a una señorita a quien se le había amputado el brazo hasta el hombro; y se sirve del brazo muy ventajosamente y no se le nota una vez vestida...". La construcción de miembros artificiales se remonta a Ambroise Paré (1510-1590) que desarrolló modelos de sorprendente complejidad ${ }^{38}$ (Fig. 4).

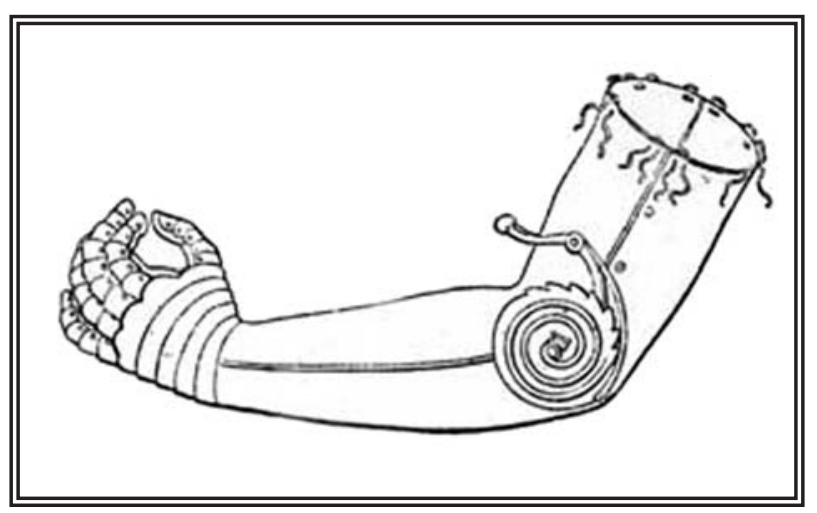

FIGURA 4. Prótesis de extremidad superior construida por Ambroise Paré.

Friedrich von Knauss (1724-1789), mecánico, relojero y cortesano, impresionó a la corte del emperador de Prusia Francisco I en 1760 con un autómata escritor, que rellenaba hojas de papel en blanco con textos a pluma, empleando un mecanismo de desplazamiento del papel e interlineado, precursor de la máquina de escribir ${ }^{39}$. En 1783 el abate Mical, presenta en la academia de París dos cabezas metálicas parlantes que logran articular palabras y pronuncian frases como “ $\mathrm{El}$ rey da la paz a Europa" y "La paz cubre al rey de gloria" ${ }^{40}$. Las cabezas parlantes constituyen un capítulo muy interesante. Ya el Papa Silvestre II ( 938 -1003), dispuso de una que contestaba "si" o "no" según fuese la pregunta ${ }^{41}$. La de Bacon, referida en párrafos anteriores, hizo que el sabio franciscano fuera llamado a capítulo por el general de la orden. También a San Alberto Magno (1206-1280) ${ }^{42}$ se le atribuye la construcción de una cabeza parlante y de un autómata que caminaba; destruído por su discípulo Santo Tomás de Aquino para librarle de la sospecha de brujería ${ }^{40}$. En España, destacan la cabeza de Tábara (pueblo de Zamora), que avisaba si había algún judío en la villa diciendo a voz en grito "judaeus adest"; o 
la que poseía el Marqués de Villena ${ }^{40}$. Uno de los episodios más curiosos del Quijote (capítulo LXII, segunda parte) trata de la "aventura de la cabeza encantada" donde se describe un cráneo metálico parlante.

En 1785 el relojero Pierre Kintzing y el ebanista David Rontgen (1743-1807), construyeron una autómata pianista que perteneció a Maria Antonieta y que se conserva aún en el museo de artes y oficios de París ${ }^{43}$. Antoine Favre, inventor de la caja de música en $1796^{44}$, aportó dos importantes conceptos de robótica: el automatismo con repetición de una tarea preprogramada; $y$ la precisión del mecanismo funcional a base de un cilindro con resaltes o de un disco giratorio con orificios. Este último dispositivo enlaza directamente con la tarjeta multiperforada del telar de Jacquard, las tarjetas de censo de Hollerith en el siglo XIX y los posteriores tarjetones de las primeras computadoras IBM.

En 1769, el ingeniero húngaro Johann Wolfgang Ritter von Kempelen (o Ján Vlk Kempelen) ${ }^{45}$ (1734-1804), construye uno de los autómatas más famosos de la historia: una máquina para jugar al ajedrez (Fig. 5). Se trataba de un dispositivo puramente mecánico, consistente en una figura humana vestida con largos

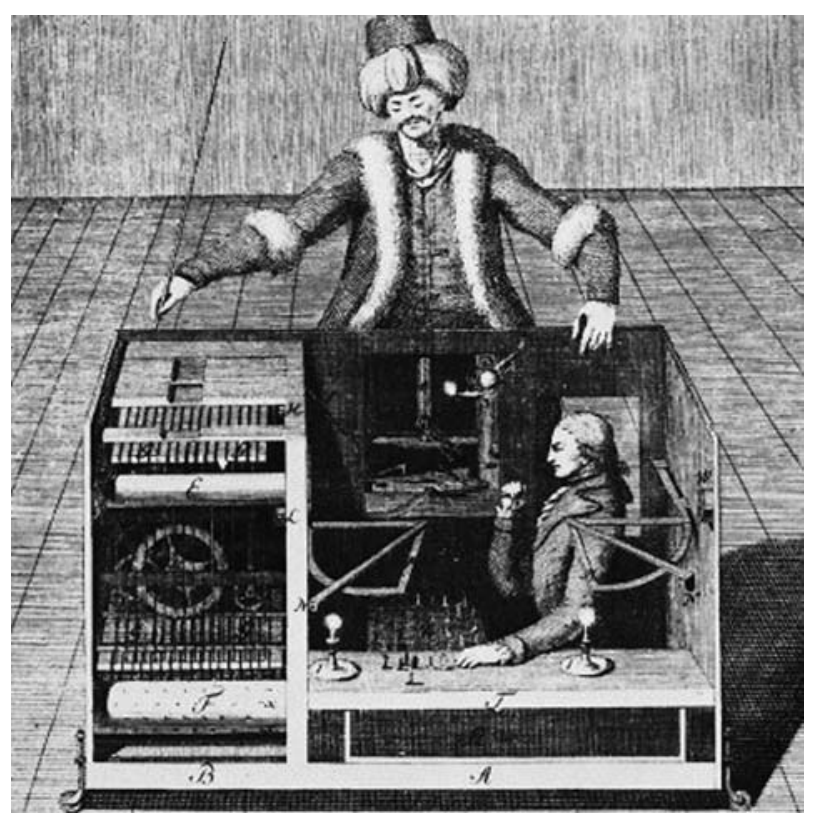

FIGURA 5. El autómata ajedrecista de Von Kempelen, en un gravado que muestra a un sujeto oculto bajo el tablero dirigiendo la partida. faldones y tocada con un turbante, sentada a una mesa de $120 \mathrm{~cm}$. de largo por $80 \mathrm{~cm}$. de alto. Por su aspecto la máquina era conocida como "el turco". Sobre la mesa había un tablero de ajedrez, y en el interior unos finos engranajes y resortes que imprimían movimiento a sus manos, que iban cambiando las fichas de posición a medida que transcurría la partida. Para demostrar que no había trucaje el inventor abría las puertas de la caja y levantaba también las largas ropas del muñeco, evidenciando que se trataba de un ingenio mecánico sin intervención humana alguna. El maniquí ganaba las partidas más complicadas y se hizo famoso en toda Europa cuando derrotó por tres veces a Napoleón Bonaparte en el palacio vienés de Schönbrunn en 1809. Se afirma que el emperador, furioso, tiró por tierra las piezas del tablero. El robot ajedrecista no respetó tampoco al emperador José II ni a la zarina Catalina II de Rusia ${ }^{46}$. Nadie consiguió descubrir el secreto de esta máquina $\mathrm{y}$, por supuesto, todos ignoraron que se habían enfrentado en realidad al campeón de ajedrez Johann Allgaier, oculto dentro del cajón ${ }^{47}$. Kempelen nunca fue descubierto y hoy día siguen siendo un misterio algunos aspectos de su invento. Además de "el turco" construyó otros ingenios, como una máquina parlante ${ }^{48}$. A fines del siglo XIX fueron construidos "Ajeeb" y "Mephisto", otros ingenios similares, dentro de los cuales se escondieron famosos ajedrecistas como Pillsbury $y$ Gunsberg ${ }^{49}$. En esta época los autómatas tenían sobre todo una intención lúdica y eran exhibidos en ferias y circos. Sólo después de unos años, a partir de la revolución industrial, empezaron a ser usados para funciones productivas.

Durante el siglo XVIII ya habían sucedido avances en el terreno industrial, como la máquina de vapor de Thomas Newcomen (1664- 1729), perfeccionada después por Humphrey Potter -que introdujo un novedoso concepto: la retroalimentación- y por James Watt (1736-1819) ${ }^{50}$. En 1801 Joseph Marie Jacquard (1752-1834) ${ }^{51}$, un empresario textil, realiza una aportación fundamental a la robótica al diseñar un sistema de funcionamiento automático de los telares, programando sus movimientos. Se trata de un cartón multiperforado que permite tipificar algunas tareas y repetirlas de forma idéntica ${ }^{52}$. Muchos años des- 
pués este sistema de programación fue incorporado por IBM en sus primeras computadoras ${ }^{53}$. En 1801, C. Spencer había inventado una máquina que producía tornillos, tuercas y arandelas de tamaño y paso variable, en función de la sustitución de guías intercambiables, que actuaban a modo de "programa"43. En 1828 el físico inglés Roben Willis construyó una máquina que pronunciaba las vocales mediante unos tubos de caña ${ }^{40}$. En 1834 el físico André-Marie Ampère (1775-1836) inicia el camino de la cibernética, estableciendo los principios de las ciencias del gobierno de máquinas. En 1898 Nicola Tesla (1856-1943), inventor del motor eléctrico de corriente alterna ${ }^{54}$, presenta el que algunos consideran el primer robot de la historia moderna, un barco teledirigido que fue presentado en el Madison Square Garden de Nueva York, a partir del que patentó el Teleautomation, un torpedo teledirigido para uso militar ${ }^{11}$. Entre finales del siglo XVIII y principios del XIX los hermanos Maillardet (Henri, Jean-David, Julien-Auguste, Jacques-Rodolphe) ${ }^{55}$ construyen un robot capaz de dibujar y escribir en inglés y francés ${ }^{56}$. Unas levas actuaban como efectores de un programa que dirigía los dispositivos responsables del proceso de escribir y dibujar ${ }^{57}$.

Jean Eugène Robert-Houdin (1805-1871) (nada que ver con Harry Hudini, famoso mago en los años 1920) ${ }^{58}$, padre de la magia moderna ${ }^{59}, \mathrm{y}$ Stèvenard, se hicieron famosos con sus autómatas. Phinéas Taylor Barnum (1810-1891) fundó el "Circo Barnum-Baily“ y el "Circo Americano" en los que reunió decenas de autómatas, algunos de ellos importados de Europa, entre los que se hallaba la máquina parlante del profesor Faber de Viena predecesor del gramófono ${ }^{40}$. En 1891 Thomas Alva Edison (1847-1931), además de sus importantes aportaciones a la técnica (lámpara incandescente, gramófono) construyó varios autómatas, entre los que destacaba una muñeca parlante. En 1906 Lee de Forest (1873-1961) desarrolla una bombilla incandescente triple conocida como tríodo ${ }^{60}$, que es una de las bases fundamentales de la circuitería electrónica moderna y que sustentó el desarrollo de las máquinas de computación hasta la invención de los transistores. Los primeros autómatas son ingenios mecánicos más o menos complicados que desarrollaban un programa fijo, pero que no empleaban necesariamente la realimentación.

Isaac Asimov (1920-1992) utilizó por primera vez el término "robótica" y postulo las tres leyes de la robótica en su libro I Robot (Yo robot) ${ }^{61}$ publicado en 1950, coincidiendo con el apogeo de la robótica moderna (Fig. 6).

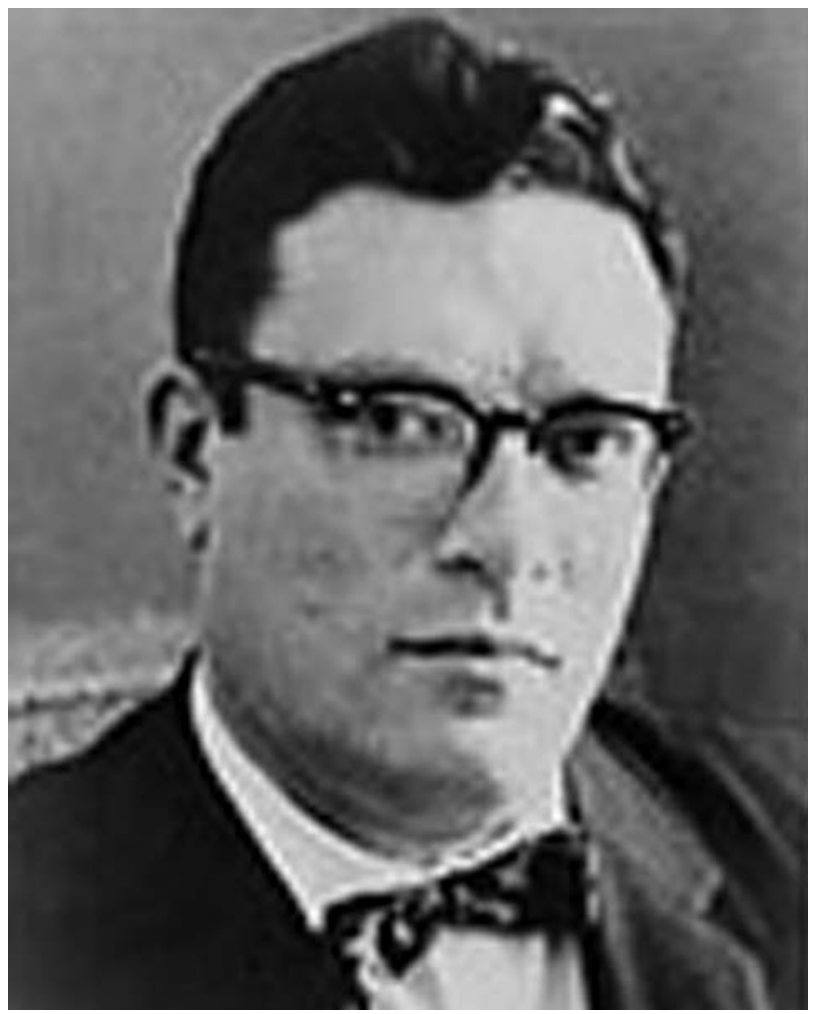

FIGURA 6. Isaac Asimov, en 1965.

\section{REFERENCIAS}

1. http://www.britannica.com/eb/article-9063935/robot

2. Karel C. R.U.R. (Rossum's Universal Robots. Penguin Classics. Referencia en http://us.penguingroup.com/nf/Book/Book Display/0,,0_9780141182087,00.html

3. Fu KS, González RC, Lee GSG. Robótica. Control, detección, visión e inteligencia. Mc Graw-Hill. Madrid. 1990.

4. http://www.monografias.com/trabajos6/larobo/larobo.shtml\# breve

5. http://en.wikipedia.org/wiki/Robot

6. http://www.vi-e.cl/internas/construy/home_constr_red.htm

7. http://www.rambal.com/educac/historia.htm

8. http://plato.stanford.edu/entries/archytas/\#date

9. http://enciclopedia.us.es/index.php/Armas_de_la_Legi\%F3n Romana

10. http://tarentum.quickseek.com/

11. http://news.bbc.co.uk/1/hi/in_depth/sci_tech/2001/artificial_intelligence/1531432.stm

12. http://archimedes.mpiwg-berlin.mpg.de/cgi-bin/toc/toc.cgi? page $=476$;dir=vitru_archi_045_it_1567;step=textonly 
13. http://www.britannica.com/ebc/article-9369466

14. http://www.brickshelf.com/gallery/inaki/Doks/euskaraz/ro botex.pdf\#search=\%22Cresibio $\% 20$ clepsidra $\% 22$

15. http://www.artehistoria.com/frames.htm?http://www.artehistoria.com/historia/personajes/4377.htm

16. http://www.britannica.com/eb/article-9003859/aeolipile

17. http://automata.cps.unizar.es/Historia/Webs/automatas_ en_la_historia.htm

18. http://www.fullborg.org/RoboticHistory.es.php?LanguageCod $\mathrm{e}=2 \&$ SectionCode $=102$

19. http://es.wikipedia.org/wiki/Aut\%C3\%B3mata_(mec\%C3\% Alnico)\#Al-Jazari

20. http://en.wikipedia.org/wiki/Strasbourg_astronomical_clock

21. http://www.geocities.com/librosmaravillosos/tecnica/perpetuum/cap01_02.html

22. http://fr.wikipedia.org/wiki/Villard_de_Honnecourt

23. http://es.wikipedia.org/wiki/Aut\%C3\%B3mata_(mec\%C3\% Alnico)\#Leonardo_Da_Vinci

24. http://www.maxmon.com/1670ad.htm

25. http://www.biografiasyvidas.com/biografia/c/caus.htm

26. http://es.wikipedia.org/wiki/Juanelo_Turriano

27. http://www.thocp.net/reference/robotics/robotics.html

28. Hassani M. Images des arts mecaniques et figure de l'ingenieur a la Reinaisance. Raport de recherche bibliographique//20022003/ENSSIB.En: http://www.enssib.fr/bibliotheque/documents/dessride/rrbhassani.pdf\#search=\%22Camus $\% 20 \% 221$ 576-1626\%22\%22.

29. http: //www.project-syndicate.org/commentary/paulbloom1/Spanish

30. Bennett S: A history of control engineering: 1800-1930. Editorial Peter Peregrinus, Londres 1979.

31. http://www.bronwenwilson.ca/physiognomy/pages/biographies.html

32. McCorduck P. Machines Who Think.: W. H. Freeman Ed. San Francisco. 1979.

33. http://www.maxmon.com/history.htm

34. http://es.wikipedia.org/wiki/Jacques_de_Vaucanson\# Carrera_como_inventor_de_aut.C3.B3matas

35. http://www.automates-anciens.com/version_espanola/frames/english_frames.htm

36. http://www.jaquet-droz.com/
37. http://www.neuchateltourisme.ch/f/villes-musees/?tb=basic \&add_id $=18 \&$ fid $=30$

38. http://www.stanford.edu/dept/HPS/representations 1.pdf\#se arch $=\% 22$ leschot $\% 20$ robot $\%$ 20prosthesis $\% 22$

39. http://es.wikipedia.org/wiki/Aut\%C3\%B3mata_(mec\%C3\% A1 nico)\#Friedrich_von_Knauss

40. http://www.ucm.es/info/museoafc/loscriminales/magiachamanismo/cabezas\%20parlentes.html

41. http://es.wikipedia.org/wiki/Aut\%C3\%B3mata_(mec\%C3\% Alnico)

42. http://es.wikipedia.org/wiki/Alberto_Magno

43. http://www.webdearde.com/modules/Tutoriales/apuntes / aprob.pdf

44. http://www.funjdiaz.net/museo/ficha.cfm?id=80

45. http://en.wikipedia.org/wiki/Wolfgang_von_Kempelen

46. http://www.portalajedrez.com/anecdotas/turco.php

47. http://es.wikipedia.org/wiki/El_Turco

48. http://www.ling.su.se/staff/hartmut/kemplne.htm

49. http://www.geocities.com/siliconvalley/lab/7378/automat.htm

50. http://es.wikipedia.org/wiki/M\%C3\%Alquina_de_vapor

51. http://www.britannica.com/ebc/article-9368382

52. http://en.wikipedia.org/wiki/Jacquard

53. http://www.maxmon.com/history.htm

54. http://en.wikipedia.org/wiki/Nikola_Tesla

55. http://en.wikipedia.org/wiki/Henri_Maillardet

56. http://www.nyu.edu/pages/linguistics/courses/v610051/gel manr/cult_hist/text/p312.html

57. http://www.fi.edu/pieces/knox/automaton/

58. http://www.geocities.com/magotrix/houdini.htm

59. http://en.wikipedia.org/wiki/Jean_Eug\%C3\%A8ne_RobertHoudin

60. http://es.wikipedia.org/wiki/Lee_De_Forest

61. Asimov I. Yo Robot. Edhasa. 1979. Barcelona.

Correspondencia autor: Dr. F.M. Sánchez-Martín

Servicio de Urología. Fundació Puigvert. Cartagena, 340-350.

08025 Barcelona. Telf. 934169700

E-mail autor: fsanchez@fundacio-puigvert.es

Información artículo: Historia de la Urología

Trabajo recibido: septiembre 2006

Trabajo aceptado: noviembre 2006 